\title{
A frozen super-Earth orbiting a star at the bottom of the main sequence ${ }^{\star}$
}

\author{
D. Kubas ${ }^{1,2}$, J. P. Beaulieu ${ }^{1,3}$, D. P. Bennett ${ }^{4}$, A. Cassan ${ }^{1}$, A. Cole ${ }^{5}$ J. Lunine 6,7 , J. B. Marquette ${ }^{1}$, S. Dong ${ }^{8,9}$, \\ A. Gould ${ }^{10}$, T. Sumi ${ }^{11}$, V. Batista ${ }^{1,10}$, P. Fouqué ${ }^{12}$, S. Brillant ${ }^{2}$, S. Dieters ${ }^{1,5}$, C. Coutures ${ }^{1}$, J. Greenhill ${ }^{5}$, \\ I. Bond ${ }^{13}$, T. Nagayama ${ }^{14}$, A. Udalski ${ }^{15}$, E. Pompei ${ }^{2}$, D. E. A. Nürnberger ${ }^{2}$, and J. B. Le Bouquin ${ }^{2}, 16$ \\ ${ }^{1}$ Institut d'Astrophysique de Paris, UMR 7095 CNRS - Université Pierre \& Marie Curie, 98 bis blv Arago, 75014 Paris, France \\ e-mail: dkubas@gmail.com \\ 2 European Southern Observatory, Casilla 19001, Vitacura 19, Santiago, Chile \\ 3 University College of London, Deparment of Physics and Astronomy, Gower Street, London, WC1E 6BT, UK \\ ${ }^{4}$ University of Notre Dame, Department of Physics, 225 Nieuwland Science Hall Notre Dame, USA \\ 5 University of Tasmania, School of Mathematics and Physics, Private Bag 37, GPO Hobart, Tas 7001, Australia \\ 6 Dipartimento di Fisica, Università degli Studi di Roma "Tor Vergata", Rome, Italy \\ 7 Department of Astronomy, 610 Space Sciences Building, Cornell University, Ithaca, NY 14853, USA \\ 8 Sagan Fellow \\ 9 Institute for Advanced Study, Einstein Drive, Princeton, NJ 08540, USA \\ 10 Department of Astronomy, Ohio State University, 140 W. 18th Ave., Columbus, OH 43210, USA \\ 11 Department of Earth and Space Science, Osaka University, Osaka 560-0043, Japan \\ 12 Observatoire Midi-Pyrénées, UMR 5572, 14 avenue Edouard Belin, 31400 Toulouse, France \\ 13 Institute of Information and Mathematical Sciences, Massey University, Private Bag 102-904, North Shore Mail Centre, Auckland, \\ New Zealand \\ 14 Department of Physics and Astrophysics, Faculty of Science, Nagoya University, Nagoya 464-8602, Japan \\ 15 Warsaw University Observatory. Al. Ujazdowskie 4, 00-478 Warszawa, Poland \\ ${ }^{16}$ Laboratoire d'Astrophysique de Grenoble, UMR 5571 Université Joseph Fourier/CNRS, BP 53, 38051 Grenoble Cedex 9, France
}

Received 28 September 2010 / Accepted 7 February 2012

\section{ABSTRACT}

\begin{abstract}
Context. Microlensing is a unique method to probe low mass exoplanets beyond the snow line. However, the scientific potential of the new microlensing planet discovery is often unfulfilled due to lack of knowledge of the properties of the lens and source stars. The discovery light curve of the super Earth MOA-2007-BLG-192Lb suffers from significant degeneracies that limit what can be inferred about its physical properties.

Aims. High resolution adaptive optics images allow us to solve this problem by resolving the microlensing target from all unrelated background stars, yielding the unique determination of magnified source and lens fluxes. This estimation permits the solution of our microlens model for the mass of the planet and its host and their physical projected separation.

Methods. We observed the microlensing event MOA-2007-BLG-192 at high angular resolution in JHKs with the NACO adaptive optics system on the VLT while the object was still amplified by a factor 1.23 and then at baseline 18 months later. We analyzed and calibrated the NACO photometry in the standard 2MASS system in order to accurately constrain the source and the lens star fluxes. Results. We detect light from the host star of MOA-2007-BLG-192Lb, which significantly reduces the uncertainties in its characteristics as compared to earlier analyses. We find that MOA-2007-BLG-192L is most likely a very low mass late type M-dwarf $\left(0.084_{-0.012}^{+0.015} M_{\odot}\right)$ at a distance of $660_{-70}^{+100}$ pc orbited by a $3.2_{-1.8}^{+5.2} M_{\oplus}$ super-Earth at $0.66_{-0.22}^{+0.51} \mathrm{AU}$. We then discuss the properties of this cold planetary system.
\end{abstract}

Key words. instrumentation: adaptive optics - stars: low-mass - planets and satellites: individual: MOA-2007-BLG-192Lb gravitational lensing: micro

\section{Introduction}

Gravitational microlensing provides a unique window on extrasolar planetary systems with sensitivity to cool planets, particularly those of low mass (Bennett \& Rhie 1996; Beaulieu et al. 2006; Gould et al. 2006; Bennett et al. 2008; Kubas et al. 2008; Sumi et al. 2010) that are currently well beyond the reach of other methods. Microlensing is also sensitive to planets orbiting

* Based on observations under ESO Prog.IDs: 279.C-5044(A) and 383-C.0495(A). very faint stars and hence spectral types not routinely examined with other techniques. In general it is a powerful tool to study the Galactic planetary population as a whole (Cassan et al. 2012).

Microlensing occurs when a foreground (lens) star passes close to the line of sight towards a background (source) star. The gravity of the foreground star acts as a magnifying lens, increasing the apparent brightness of the background star as it gets close to the line of sight. A planetary companion to the lens star will induce a perturbation to the microlensing light curve with a duration that scales with the square root of the planet mass, 
lasting typically a few hours for an Earth to a few days for a Jupiter (Gould \& Loeb 1992; Mao \& Paczynski 1991; Liebes 1964). Hence planets are now routinely discovered by dense photometric sampling of ongoing microlensing events. The inverse problem, finding the properties of the lensing system from an observed light curve, is a complex non-linear one within a wide parameter space. The planet/star mass ratio and projected star-planet separation can usually be measured with high precision. However in the absence of higher order effects such as parallax motion and/or extended source effects, in general there are no direct constraints on the physical masses and orbits of the planetary system. In the least information case, model distributions for the spatial mass density of the Milky Way, the velocity distribution of potential lens and source stars, and the mass function of the lens stars are used in a Bayesian analysis to derive probability distributions for the masses of the planet and the lens star and their distance, as well as the orbital radius and period of the planet.

With complementary high angular resolution observations, currently done either by HST or with adaptive optics, it is possible to get additional strong constraints on the system parameters and determine masses to about $10 \%$. This can be done by directly measuring the light coming from the lens and measuring the lens and source relative proper motion (Bennett et al. 2006, 2007, 2010; Gaudi et al. 2008; Dong et al. 2009; Janczak et al. 2010)

An extrasolar planet with a best-fit mass ratio of $q \sim 2 \times 10^{-4}$ was discovered in the microlensing event MOA 2007-BLG-192 (Bennett et al. 2008) found by the MOA collaboration toward the Galactic bulge, (J2000: RA, Dec $)=(18: 08: 03.8,-27: 09: 00)$. The best fit microlensing model shows both microlensing parallax and finite source effects. Combining these, we obtained the lens masses of $M_{1}=0.06_{-0.021}^{+0.028} M_{\odot}$ for the primary and $3.3_{-1.6}^{+4.9} M_{\oplus}$ for the planet. The incomplete light curve coverage of the planetary anomaly led to a significant degeneracy in the lens models, and the lack of strong constraints on the source size to a poorly determined Einstein radius. Together this resulted in rather large uncertainties in the physical parameter estimates of the system.

Additional constraints are required to exclude competing microlens solutions and to refine our knowledge of the physical parameters of the system. It is possible to constrain masses and parameters of the system thanks to high angular resolution imaging. Most microlensing events only provide a single parameter, the Einstein ring crossing time $t_{\mathrm{E}}$, that depends on the mass of the lens system $M_{\mathrm{L}}$, its distance $D_{\mathrm{L}}$, the source distance $D_{\mathrm{S}}$ and their relative velocity. However, when the relative lens-source proper motion $\mu_{\text {rel }}$ can be determined this yields the angular Einstein ring radius $\theta_{\mathrm{E}}=\mu_{\text {rel }} t_{\mathrm{E}}$. Moreover $\theta_{\mathrm{E}}$ is linked to the lens system mass by

$M_{\mathrm{L}}=\frac{c^{2}}{4 G} \theta_{\mathrm{E}}^{2} \frac{D_{\mathrm{S}} D_{\mathrm{L}}}{D_{\mathrm{S}}-D_{\mathrm{L}}}$,

therefore, since the distance of the source $D_{\mathrm{S}}$ is known from its magnitude and colors, Eq. (1) is a mass-distance relation for the lens star. Another constraint is needed to obtain a complete solution to the microlensing event. This can be achieved by directly detecting light from the planetary host star (the lens). Combining this measurement with Eq. (1) and a mass luminosity relation will yield the mass of the lens. This has been done already for several microlensing events where the system is composed of a star and a gaseous planet (Bond et al. 2004; Bennett et al. 2006; Udalski et al. 2005; Dong et al. 2009; Gaudi et al. 2008; Janczak et al. 2010).
Table 1. Log of JHKs NACO data.

\begin{tabular}{lcccc}
\hline \hline Band & $n \times \operatorname{Exp}[\mathrm{s}]$ & MJD & Airmass & FWHM ["] \\
\hline \multicolumn{5}{c}{ epoch 1 } \\
$J$ & $6 \times 60$ & 54350.00781250 & 1.005 & 0.14 \\
$H$ & $20 \times 25$ & 54350.02734375 & 1.023 & 0.19 \\
$K \mathrm{~s}$ & $10 \times 25$ & 54349.98828125 & 1.002 & 0.09 \\
\hline \multicolumn{5}{c}{ epoch 2 } \\
$J$ & $23 \times 60$ & 55036.08593750 & 1.015 & 0.34 \\
$H$ & $22 \times 30$ & 55036.06640625 & 1.034 & 0.29 \\
$K \mathrm{~s}$ & $24 \times 30$ & 55015.10156250 & 1.088 & 0.10 \\
\hline
\end{tabular}

Notes. According to the Paranal night logs the epoch 1 night was classified as photometric, whereas the epoch 2 observations were taken in clear sky condition. We give the exposure time, modified Julian date, airmass and measured full width at half max on the coadded frames.

We observed MOA-2007-BLG-192 in JHK using adaptive optics on the VLT while it was still amplified by a factor of 1.23 and again when the microlensing was over. Here, we combine the NACO $J H K$ flux measurements at these 2 epochs with the color estimate of the source star (Gould et al. 2010) and the microlensing model (Bennett et al. 2008) to disentangle the flux coming from the source and from the lens star to refine estimates of the parameters of the system.

\section{The data set}

We obtained JHKs measurements using the NACO AO system (Lenzen et al. 2003; Rousset et al. 2003) mounted on Yepun during the night 6/7 Sept. 2007, while the source star was still magnified by a factor of 1.23 . AO corrections were performed on a natural guide $\operatorname{star}^{1}$ and observations with the S27 objective $\left(27^{\prime \prime} \times 27^{\prime \prime}\right.$ FOV, pixelscale $\left.=0.02715^{\prime \prime}\right)$ were conducted in jitter mode with multiple exposures at random offsets within $10^{\prime \prime}$ of the target. In the absence of suitable "empty" sky patch close to the target, this strategy was chosen to ensure an accurate estimation of the sky background and to filter out bad pixels. The second epoch(s) were obtained with the same observing strategy more than 22 months later with the event being at baseline, i.e. when the source was not magnified anymore. An overview of the NACO data set is given in Table 1 .

To perform absolute calibration of the NACO images we obtained $90 \times 10 \mathrm{~s}$ dithered images in JHKs of the MOA-2007-BLG-192 field with the Sirius simultaneous 3-band camera (Nagayama et al. 2003) on the Japanese/South African IRSF 1.4 m telescope (Glass \& Nagata 2000) at SAAO (non AO, $\sim 8^{\prime} \times 8^{\prime}$ FOV, pixelscale $\left.=0.45^{\prime \prime}\right)$ on 29th of Aug. 2008, i.e. at a time when the event was at baseline.

\subsection{Reduction}

Following a "lucky imaging" approach we visually inspect each of the NACO raw images and remove the ones for which the AO correction was obviously poor. The remaining raw frames are then dark-subtracted with darks of exposure times matching the science frames, flatfielded with skyflats, median co-added and sky-subtracted using recipes from the Jitter/Eclipse infrared data

\footnotetext{
1 The LGSF, which in theory should have yielded better performance, was not available at that time. However the pro and contra of LGS vs. NGS for us have to be evaluated on a case by case base, since in the crowded field of microlensing targets one often finds suitable NGS references which may give even better corrections than the LGSF according to the ETC observation preparation software.
} 


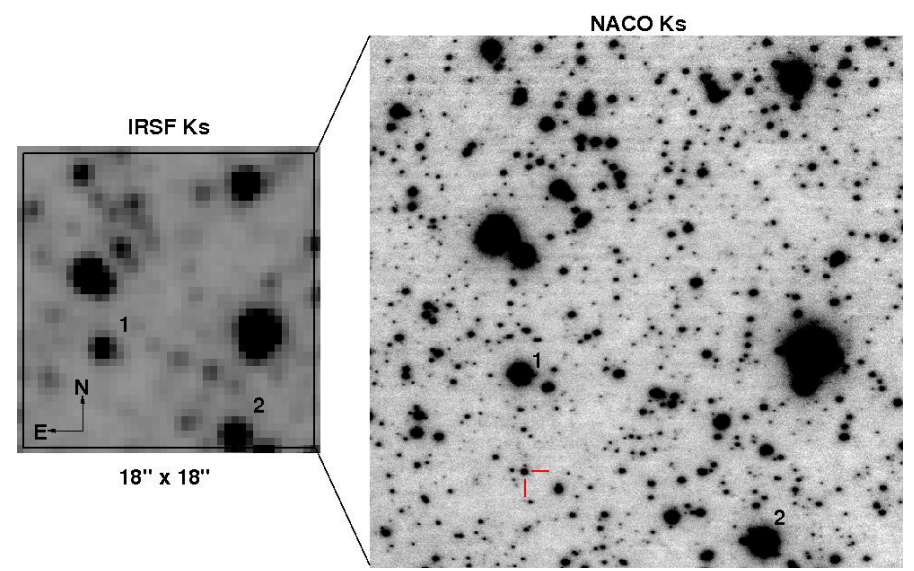

Fig. 1. Left: extract of IRSF Ks band image of MOA-2007-BLG192 used to calibrate the NACO photometry of the $18^{\prime \prime} \times 18^{\prime \prime}$ large intersection fov of the coadded NACO frames in $K \mathrm{~s}$ band (right). MOA2007-BLG-192 is marked with the half cross hair. The stars annotated with " 1 " and " 2 " serve as PSF-reference and photometric zeropoint calibrators. Furthermore these two stars are common to all bands and epochs. The bright stars north of the two references are either too crowded, in the non-linear regime or too far away from the target.

reduction package by Devillard (1997, 1999). To avoid border effects, we keep only the intersection of the different dither positions of the co-added frames for our photometric analysis.

The IRSF data, which was taken to gauge our NACO data, has been dark-subtracted, flat-fielded and sky subtracted using the on-the-mountain pipeline package for the SIRIUS camera (Nagayama et al. 2003).

\section{Photometric analysis}

As in our previous analysis of planetary microlensing event MOA-2008-BLG-310Lb (Janczak et al. 2010), we extract the photometry of NACO images using Starfinder (Diolaiti et al. 2000). This tool is tailor suited to perform photometry of AO images of crowded fields. It creates a numerical PSF template from chosen stars within the frame, which is then used for PSF-fitting of all stars in the field. To build our PSF reference we chose the star marked as " 1 " in Fig. 1 based on the following criteria. It is close to the target (within less than $4^{\prime \prime}$ ), sufficiently bright but well within the linearity regime of the detector and common to all final reduced $J H K$ s images of both epochs. Figure 6 shows the $J H K$ s images centered on the target for the first epoch of NACO and the PSF subtracted residuals. The IRSF photometry catalog was created with DoPhot (Schechter et al. 1993).

\subsection{Building a calibration ladder}

In order to build the calibration ladder, we use three data sets: the 2MASS catalogue, photometry obtained at the IRSF telescope and then NACO data. It is necessary to use the intermediate step of IRSF observations because we have too few stars in common between 2MASS and NACO. Such ladder has been used already and described in the appendix of (Janczak et al. 2010). The three $J H K$ s color systems are very close. For example, the color term for $J-K$ s colors between 2MASS and IRSF is only 0.01 . Accurate calibration between 2MASS and IRSF has been given in Kato et al. (2007). Janczak et al. (2010) did not detect color terms between NACO and IRSF filters. Therefore, our calibration ladder must only determine the zeropoint offsets.
Table 2. JHKs NACO photometry for MOA-2007-BLG-192, i.e. lens+source (no dereddening applied).

\begin{tabular}{lccc}
\hline \hline Band & $J$ & $H$ & $K_{\mathrm{S}}$ \\
\hline \multicolumn{4}{c}{ NACO epoch 1 } \\
\hline calibrated against IRSF \\
$19.209 \pm 0.043$ & $18.281 \pm 0.042$ & $17.948 \pm 0.035$ \\
\hline \multicolumn{4}{c}{ NACO epoch 2 } \\
\hline calibrated against IRSF & $18.324 \pm 0.073$ & $18.548 \pm 0.112$ & $17.989 \pm 0.038$ \\
$\Delta$ epochs & $0.115 \pm 0.085$ & $0.267 \pm 0.120$ & $0.041 \pm 0.052$ \\
\hline aligned with respect to epoch 1 \\
\multicolumn{5}{c}{$19.283 \pm 0.071$} & $18.498 \pm 0.087$ & $18.011 \pm 0.042$ \\
$\Delta$ epochs & $0.074 \pm 0.083$ & $0.217 \pm 0.097$ & $0.063 \pm 0.055$ \\
\hline
\end{tabular}

Notes. The absolute photometry error budget is composed by adding in quadrature the errors on the zeropoint, the formal error reported by Starfinder and the background error as estimated from the scatter between epoch 1 and epoch 2 comparison stars. For epoch $1, J$, and $H$ bands, we adopt the background error estimate as derived from the $K$ band, since the poor epoch 2 quality in $J$ and $H$ would overestimate the epoch 1 errors.

We first perform the astrometry of the IRSF images with respect to the online 2MASS catalog using GAIA/Skycat and WCSTools. Then, using only stars marked as AAA (highest 2MASS quality flag) in the JHKs bands we crossmatch the common stars to compute the photometric transformation between the two catalogs by sigma clipping, demanding an astrometric accuracy of the match of better than $0.6^{\prime \prime}$. To minimize the effect of source confusion and blending contamination we cut off at magnitude 13 for the 2MASS reference stars and sum up the flux of close neighbors for the IRSF sources to account for the much coarser pixel scale of the 2MASS catalog.

The PSF reference star is contained in the IRSF catalog, as well as star "2" (Fig. 1). We examine their long term photometric stability in the OGLE database and find that over more than seven years both stars are stable (in the optical $I$-band) at levels of $\lesssim 1 \%$, which makes them well suited as zeropoint calibrators of our NACO field. While we adopt star " 1 " as the primary photometric calibrator since star " 2 " is more crowded, we determine zeropoints from both stars as a consistency check. To account for the different plate scales between NACO and IRSF we sum up the flux of all the NACO sources which are contained within the IRSF PSF. We note that observing conditions (sky transparency and atmospheric coherence times) for the second epoch data set were inferior to the epoch 1 measurements and the uncertainties in the absolute zeropoints of epoch 2 are therefore larger. Since we are mainly interested however in the relative photometry of the two epochs we can align the epoch 2 photometry with respect to more accurately calibrated epoch 1. Table A.1 summarizes this way of determining the transformations to calibrate the NACO data with respect to the 2MASS system and Table 2 shows our derived photometry for MOA-2007-BLG-192.

\section{Results}

In Figs. 2 and 3 we present the color-magnitude diagrams for the combined IRSF and NACO (epoch 1) data. To estimate the interstellar extinction, we first determine the position of the red clump center by taking the median of the distributions in color and magnitude inside a window centered on a first guess estimated position. Then we fit the tip of the Red Giant Branch as given by the isochrones of Marigo et al. (2008) adopting the distance modulus $\mathrm{dm}=14.38 \pm 0.07$ as found for the 

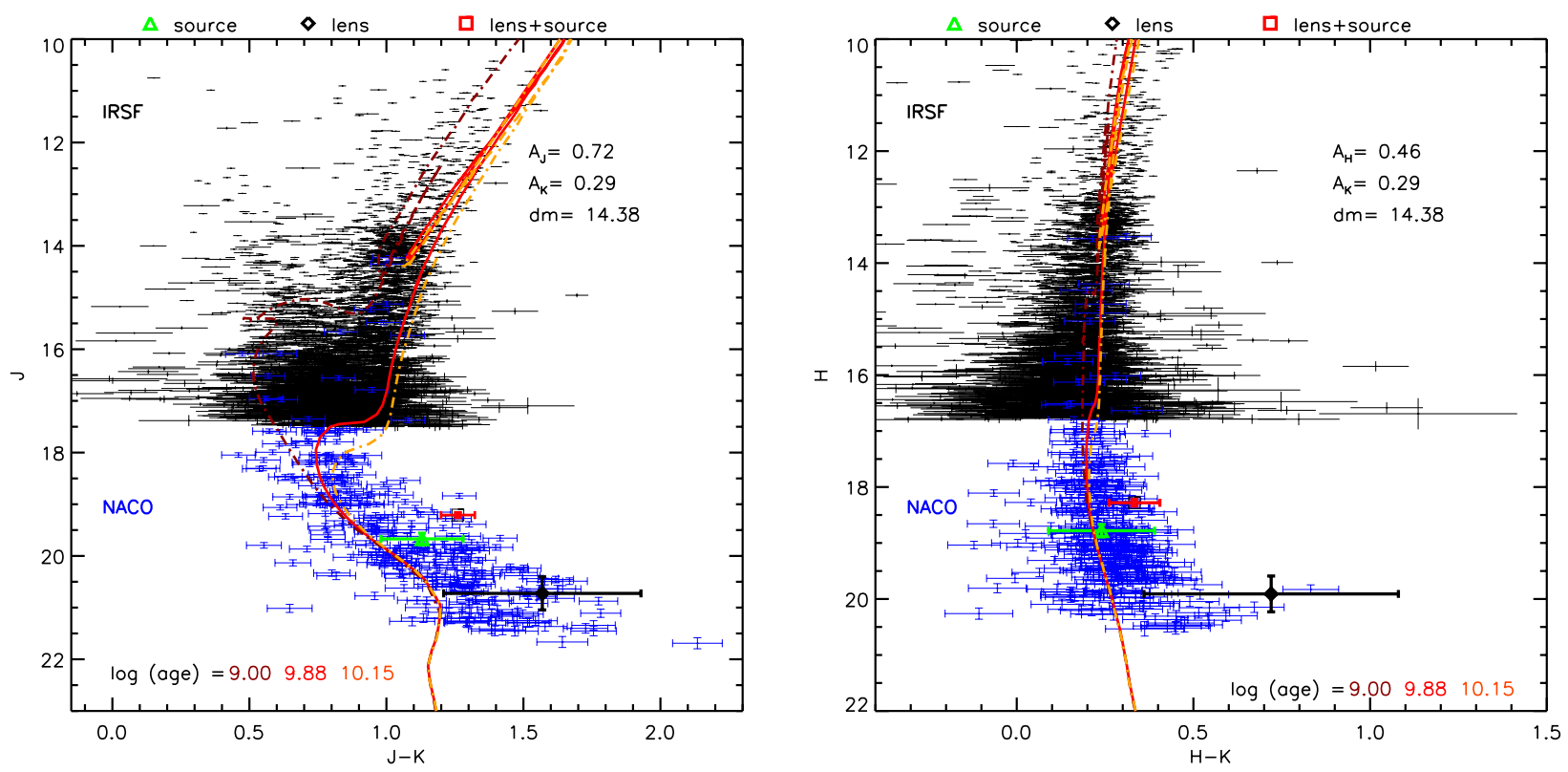

Fig. 2. Left: the $(J-K \mathrm{~s}, J)$ CMD in the 2MASS system of the MOA-2007-BLG-192 field combining the data from the IRSF (within $3^{\prime}$ of target, black points) and NACO (within $18^{\prime \prime}$, blue points). In red the photometry of the measured lens+source flux at magnification $A=1.23$ is displayed together with the inferred decomposed fluxes of the source (green) and the lens (planetary host star, black). Overplotted are Marigo et al. (2008) solar metallicity isochrones of ages $\log (\mathrm{Gyr})=9.00,9.88,10.15$ at distance modulus of $\mathrm{dm}=14.38$ and estimated extinction of $A_{J}=0.72, A_{K s}=0.29$. Right: same as above but for $(H-K \mathrm{~s}, H)$.

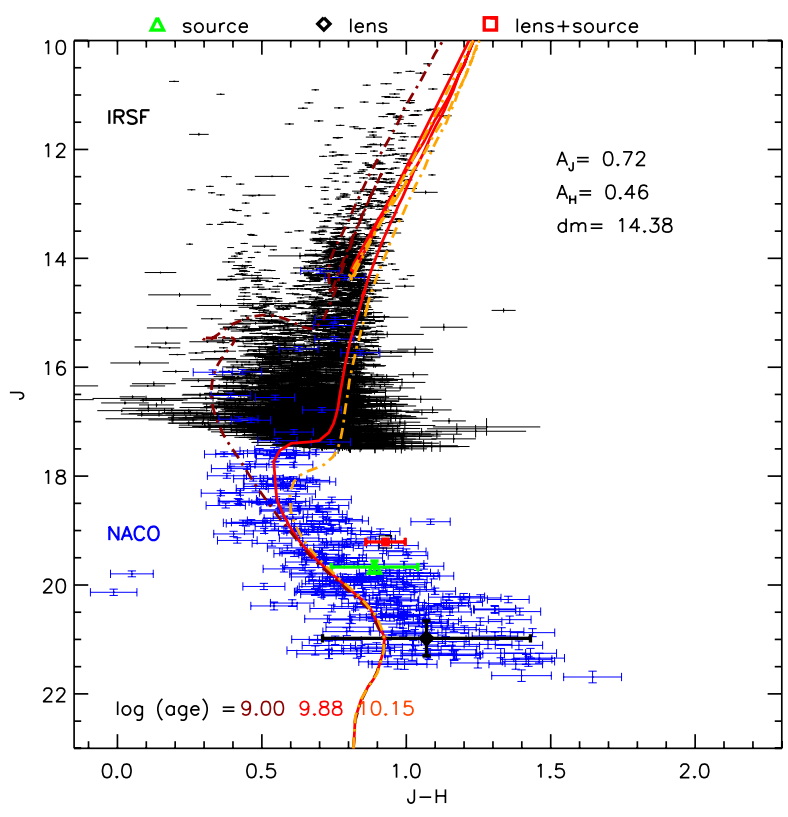

Fig. 3. Same as Fig. 2 but for $(J-H, J)$.

MOA-2007-BLG-192 field by Bennett et al. (2008). With a best fit age of $\log (\mathrm{Gr})=9.88$ we find for the extinction coefficients: $A_{J}=0.72 \pm 0.10, A_{H}=0.46 \pm 0.10, A_{K}=0.29 \pm 0.10$, which for this line of sight is consistent with extinction maps from Schlegel et al. (1998) and Marshall et al. (2006).

\subsection{The case for a luminous lens I: NACO-only}

The standard general microlens light curve model is given as

$$
F(t)=F_{\mathrm{S}} A(t)+F_{\mathrm{B}},
$$

where $F$ is the measured flux at the telescope, $F_{\mathrm{S}}$ is the intrinsic unmagnified source flux, $A(t)$ the time dependent magnification given by the lens model and the blend flux $F_{\mathrm{B}}=F_{\mathrm{L}}+F_{\text {Background }}$, which contains the lens flux $F_{\mathrm{L}}$ and $F_{\text {Background }}$ the flux of any unrelated field stars within the aperture, $F_{\text {Background. While the }}$ source flux $F_{\mathrm{S}}$ can be determined with high precision from the light curve modeling of the non-AO data, given a large magnification gradient, the background term normally dominates over the lens term in seeing-limited photometry of typically crowded Galactic Bulge fields of microlensing. Hence the benefits of high spatial resolution imaging are obvious. Reducing or eliminating the contribution of contaminating background sources provides a better estimate of the lens flux and so finally of the physical characteristics of the lens system. In Janczak et al. (2010) the lens flux could be estimated by comparing a single NACO AO epoch with an excellent seeing-limited light curve in the same passband from which the source flux had been previously determined with good accuracy. For MOA-2007-BLG-192Lb we have no such light curve in the NACO passbands but a well determined measurement of the source flux in the $I$ band (Cousins system), which we can transform into the expected source flux for $J H K$ s bands. Note that while in theory our two point NACO "light curve" can be used to solve Eq. (2) for the lens and source fluxes directly, the resulting uncertainties are very large (Dong et al. 2009) due to very small magnification "lever arm" for our event and so following the path of Janczak et al. (2010) is much more accurate.

First, however, the two epochs can be used as follows, without the knowledge of the source flux, to check whether there is an indication that light from the lens is detected. The expected magnification gradient between the two NACO epochs based on the best-fit model of Bennett et al. (2008) is $\Delta m=$ $0.230 \pm 0.015 \mathrm{mag}$. Note that this gradient is basically the same for all competing planetary models, since the first epoch was taken close to the baseline of the event, where the single lens approximation describes the data very well. If the lens is dark and no unrelated source is contaminating our photometry (see Sect. 4.3) we then would expect to measure this difference in the 

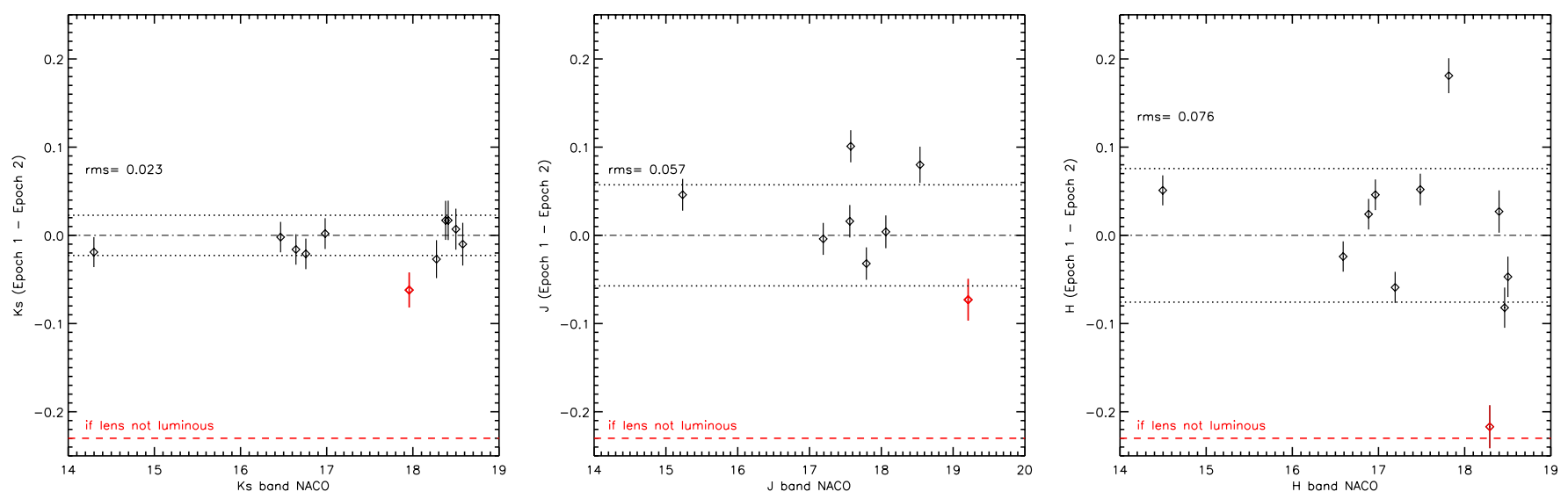

Fig. 4. Relative photometry of the NACO epochs for each band calibrated/aligned to epoch 1 based on stars within 4" of MOA-2007-BLG-192. The black points are constant stars, so their scatter gives an estimate of the error. The red dashed line marks the expected magnitude difference of the target assuming no light from the lens is detected. The red diamonds show the flux changes of the target, which are inconsistent with such a scenario at a $3-\sigma$ level for $J$ and even with higher significance for our best data set in $K$ band. The poor quality of the epoch $2 H$ band data as evident by the large scatter does not provide any useful constraint. Table 2 gives the derived values for all bands and different choices of the field of comparison stars.

relative photometry of the two epochs in each band. Since the quality of epoch 1 is superior we choose epoch 1 as reference to which we align epoch 2 . We compare the photometry between the two epochs for each band using 3 different alignment procedures. First we compare the derived absolute photometry (with respect to 2MASS using the calibration ladder described in Sect. 3.1).

Then we align epoch 2 with respect to (calibrated) epoch 1 using all common stars within 4 " (to minimize effect of PSF variations) of the target. The resulting magnitude differences for the target and the absolute photometry values are summarized in Table 2. The difference between the epochs is shown in Fig. 4. Regardless of the alignment method used, for all bands except $H$ (the set with the poorest epoch 2 data quality), the measured difference is less than in the case of a dark lens, albeit with different levels of significance. For $K$ band, the best data set, a dark lens is inconsistent with the measurement at $2 \sigma$ for the absolute alignment and at $3 \sigma$ for the relative alignment. The results for $J$ band are also inconsistent with a dark lens, but in this case at slightly less than $2 \sigma$. This motivates a more careful examination of the evidence for a luminous lens.

\subsection{The case for a luminous lens II: NACO+IRSF+optical}

A more powerful test for the presence of "excess light" (in addition to that of the source) is possible by combining NACO, IRSF, and optical data. To maximize sensitivity, we will work entirely with uncalibrated data. This will eliminate any errors associated with calibration relations, extinction estimates, and color-color relations. The remaining errors, which are either measurement errors or intrinsic scatter, are both small and easy to measure.

We begin by constructing a color-+color diagram that combines optical $V$ and $I$ data from OGLE-II with $K$ data from IRSF (see Fig. 5). The OGLE-II data are used because this is the system in which Gould et al. (2010) measured the color and magnitude of MOA-2007-BLG-192S,

$(V-I)_{\mathrm{s}}=2.36 \pm 0.03 ; \quad I_{\mathrm{S}}=21.45 \pm 0.05$.

The OGLE-II data are in fact calibrated, but that is incidental: the important thing is that the optical color is measured in this
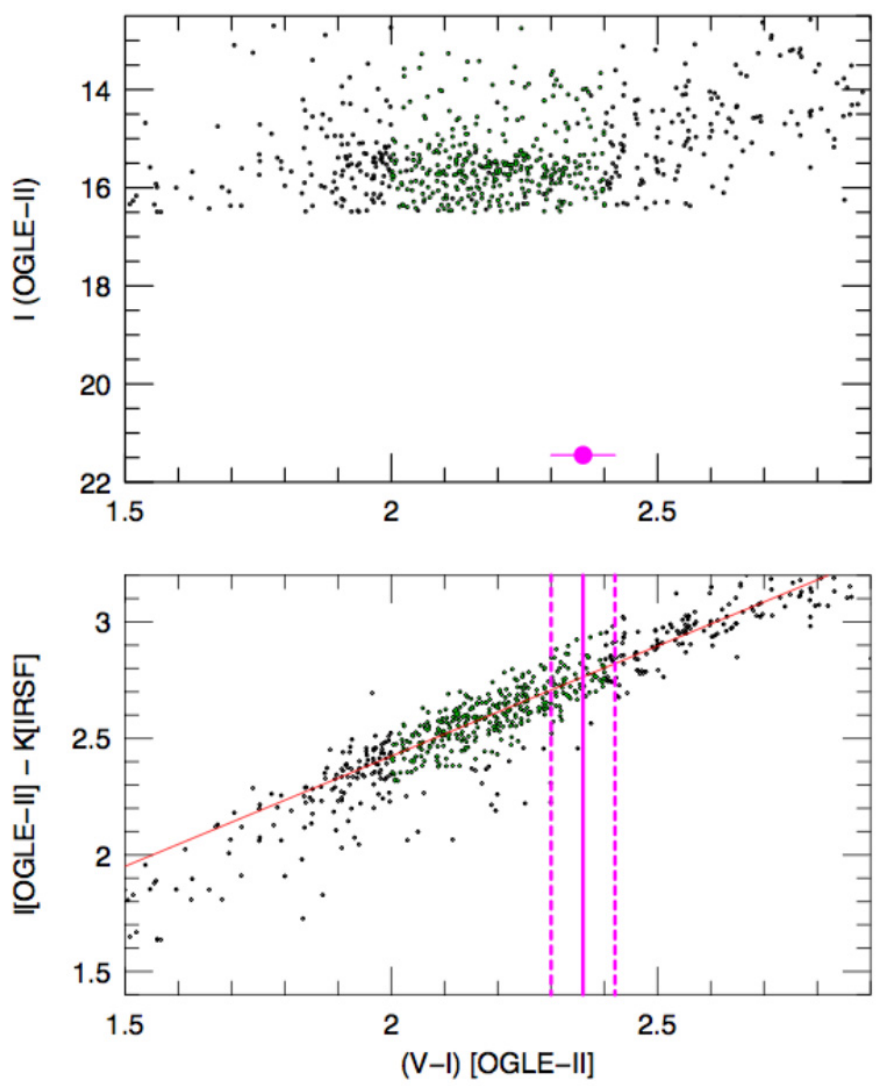

Fig. 5. Empirical VIK instrumental color-color relation. Lower panel: open black symbols show all astrometric matches (that survived a strict crowding criterion) between OGLE-II $V / I$ data and instrumental IRSF $K$ data. The green filled points show those used in the fit (red line), which are restricted to $2.0<(V-I)<2.4$ and exclude $3 \sigma$ outliers. Vertical magenta lines show MOA-2007-BLG-192S $(V-I)$ color measurement by Gould et al. (2010). Upper panel shows the VI CMD positions of stars used to determine the VIK relation. They are all giants in or near the clump, whereas the source (magenta point) is a dwarf. Nevertheless, Bessell \& Brett (1988) show that the VIK relations are essentially identical for giants and dwarfs in this color range (see text). 
system. The $K$ magnitudes are constructed directly from IRSF photometry fluxes $K=22.155-2.5 \log$ (flux). The zero-point constant is chosen for convenience to be similar to the calibration constant, but this constant does not enter the calculation in any way. In particular, the data remain uncalibrated because there is no color term.

The open black circles are all the astrometric matches that meet a strict crowding criterion. A color-color relation (red) is derived by fitting the points in the range $2.0<(V-I)<2.4$ with $3 \sigma$ rejection (green filled points). This choice of interval will be justified below. The relation is:

$(I-K)=Z_{1}+Z_{2}[(V-I)-2.36]$

$Z_{1}=2.757 \pm 0.008 ; \quad Z_{2}=0.943 \pm 0.039$

with a scatter of 0.080 mag.

The vertical lines represent the best fit and error bar of the Gould et al. (2010) optical-color measurement. From Eqs. (3) and (4), the best estimate of the source magnitude at the first epoch (when the source was magnified by $A=1.23$ ) is therefore

$$
K_{\mathrm{s}}=\left[I_{\mathrm{s}}-2.5 \log (A)\right]-Z_{1}-Z_{2}\left[(V-I)_{\mathrm{s}}-2.36\right]=18.468 .
$$

We discuss all the errors in this estimate below.

We now compare this with the baseline flux as measured by NACO and transformed to the IRSF system:

$K_{\text {base }, \mathrm{IRSF}}=K_{\text {comp,IRSF }}+2.5 \log \left(F_{\text {comp,NACO }} / F_{\text {base, } \mathrm{NACO}}\right)=17.948$

where $K_{\text {comp,IRSF }}=14.261 \pm 0.016$ is the IRSF magnitude of the comparison star, and $F_{\text {comp,NACO }}=88066 \pm 37$ and $F_{\text {base, NACO }}=$ $2952.2 \pm 12.1$ are the NACO fluxes of the comparison and baseline stars, respectively.

The baseline flux is clearly larger, $K_{\mathrm{s}}-K_{\text {base }}=0.520$, The question is, how large is the error in this difference? Equation (5) has 5 identifiable sources of error. First, the error in $I_{\mathrm{s}}$ is 0.05 , but the error in $\left[I_{\mathrm{S}}-2.5 \log (A)\right]$ is actually smaller than this by a factor 0.57 (see Eq. (10) of Janczak et al. 2010). Second, the error in $Z_{1}$ is 0.008 . Third the error in the final term is $Z_{2}$ times the error in $(V-I)_{\text {s }}$, i.e., 0.056 .

Fourth, we are using the $V I K$ color-color relation of the field stars observed by IRSF as a proxy for the VIK color-color relation of the source. However, the source is a dwarf, while the field stars are all giants. Now, according to Fig. 1 of Bessell \& Brett (1988) these $V I K$ relations of giants and dwarfs are virtually identical for $(V-K)_{0}<3.0\left[(V-I)_{0}<1.3\right]$, and diverge only very slowly at redder colors. The dereddened color of the source is $(V-I)_{0}=1.24 \pm 0.06$, so the entire $1 \sigma$ error range lies within the "same relation" region. And again, the relations diverge only very slowly at redder colors. We note, however, from Fig. 2 of Bessell \& Brett (1988), that the divergence is extremely rapid in $(J-K)$. This is the principal reason that we conduct this test in $K$ rather than $J$.

Finally, the $V I K$ relation in Fig. 5 exhibits a scatter of $0.08 \mathrm{mag}$. If this scatter is attributed to measurement errors, then the effect is very small. There is some reason to expect that this is the case because Fig. 1 of Bessell \& Brett (1988) shows almost zero scatter. However, the bulge star population may be more diverse than the local one. For the moment we assume it is intrinsic, and that this scatter in the observed giant VIK relation also applies to dwarfs. Then, the error in $K_{\mathrm{s}}(A=1.23)$ is $\left[0.028^{2}+0.008^{2}+0.056^{2}+0.08^{2}\right]^{1 / 2}=0.102$.

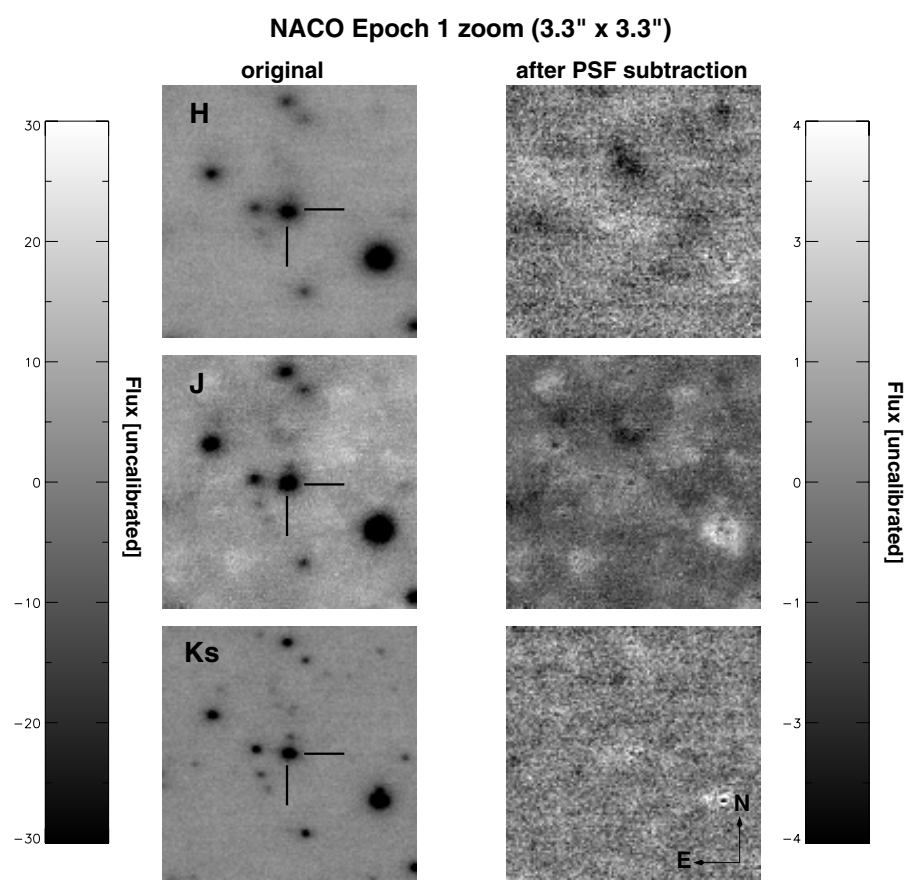

Fig. 6. Left: zoom $\left(3.3^{\prime \prime} \times 3.3^{\prime \prime}\right)$ of epoch 1 NACO images centered on the target in $H$ (top), $J$ (middle), and $K$ (bottom). Right: images after PSF subtraction. The PSF subtraction does a good job of removing essentially all flux, particularly in $K$.

There are three errors contributing to $K_{\text {base, }}$, which are listed below Eq. (6). Their sum in quadrature is 0.017. As seen in section 3 the JHKs bands from 2MASS, IRSF and NACO are very close, with negligible color terms in the transformations between the different systems. Next we note that photometry on AO images is notoriously difficult due to gradients in the PSF. This effect is hard to quantify. However, Fig. 6 shows that it must be quite small. The left (right) column shows the image before (after) PSF subtraction for the first epoch, for $J, H, K$, respectively. The $K$ image in particular, looks extremely clean. We nevertheless conservatively estimate a 0.03 photometry error due to PSF gradient. This yields a total error on $K_{\text {base }}$ of $0.034 \mathrm{mag}$.

Finally, we note that even if the scatter in Fig. 5 were due to measurement noise, rather than intrinsic scatter (and so should not have been included in the error in $K_{\mathrm{s}}(A=1.23)$, it would then contribute to the error in $K_{\text {base }}$ through $K_{\text {comp,IRSF. Hence, }}$ the impact on the final error would have been identical.

We therefore finally derive our estimate of the excess magnitude at baseline:

$K_{\mathrm{s}}-K_{\text {base }}=0.520 \pm 0.108$

which is a $4.8 \sigma$ detection of additional light. It is either a blend aligned to better than 0.1 arcsec with the source star of the microlensing event or light coming from the lens star.

\subsection{Is the blended light from the lens star?}

The mean density of stars of comparable brightness and color $\pm 0.20 \mathrm{mag}$ to the detected blend is less than 0.2 per $\operatorname{arcsec}^{2}$ as derived from our best/sharpest data set, the $K$ s band of epoch 1 . Given the image quality of $0.09^{\prime \prime}$ FWHM, this conservatively implies a probability of less than $2 \%$ for the blend being unrelated to the microlens event. Another possibility to consider is 
that the blend stems from a companion to the source star. Close companions with periods $\lesssim 100 \mathrm{~d}$ can be ruled out by the xallarap signal limits in the light curve and very wide separation companions $\gtrsim 700 \mathrm{AU}$ would be resolved in the Ks NACO data. This still leaves a large range of allowed separations but taking into account the color difference the possible fraction of low mass secondaries should not be larger then $8 \%$ according to Duquennoy \& Mayor (1991). However only future AO or HST images, when the source and lens will have moved sufficiently far apart to be spatially resolved, will be able to securely rule out such a scenario.

\subsection{Source star constraints}

To compare the $(V-I)_{0}=1.24 \pm 0.06$ color found by Gould et al. (2010) to the NIR bands of this study we transform this $V-I$ color to $J-K$ in the 2MASS system using first the dwarf color table of Bessell \& Brett (1988) to find $(J-K)_{0, \mathrm{~s}}=0.73$ and then with the 2MASS-Bessell \& Brett filter relation ${ }^{2}$ we finally derive $(J-K)_{0, \mathrm{~s}}=0.70 \pm 0.07$.

From our NACO "light curve" using Eq. (2) and our lens model, we find after dereddening $(J-K)_{0, \mathrm{~s}}=0.66 \pm 0.51$. While the uncertainty derived from linear regression is large, this independent source color determination is very consistent with the colors found by Gould et al. (2010) as well as those of Bennett et al. (2008) and strengthens the case for the source being a K4-5 dwarf in the Bulge at $7.51 \pm 0.25 \mathrm{kpc}$. However, given the better accuracy of the Gould et al. (2010) source color, we adopt their value in the following analysis.

\subsection{Lens/planetary system constraints}

From the MOA-2007-BLG-192 light curve the $I$ band source flux is well determined to $I_{\mathrm{s}}=21.44 \pm 0.08$ (Bennett et al. 2008). Using the source color derived in the previous section and the extinction coefficients determined from the IRSF data, we can translate this $I$ band estimate into the NACO passbands to derive $J_{\mathrm{s}}=19.67 \pm 0.12, H_{\mathrm{s}}=18.78 \pm 0.10, K_{\mathrm{s}}=18.54 \pm 0.10(2 \mathrm{MASS}$ system).

Using our best lens model and Eq. (2) we then derive the following estimates for the apparent lens flux: $J_{1}=20.98 \pm$ $0.30, H_{1}=19.91 \pm 0.30, K_{1}=19.29 \pm 0.20$ from epoch 1 and $J_{1}=20.59 \pm 0.40, H_{1}=20.10 \pm 0.50, K_{1}=19.04 \pm 0.20$ from epoch 2 . Taking the weighted average we finally get as best estimate for the lens flux: $J_{1}=20.73 \pm 0.32, H_{1}=19.94 \pm 0.35, K_{1}=$ $19.16 \pm 0.20$.

We now can use mass luminosity relations to translate the photometry estimates of the apparent lens flux into estimates of the planetary host star mass. We adopt the relations of Delfosse et al. (2000) for M-dwarfs (with masses $>0.10 M_{\odot}$ ) and Chabrier et al. (2000) for L-dwarfs (masses $<0.10 M_{\odot}$ ), where the transition between the two relations at $\sim 0.10 M_{\odot}$ has been linearly interpolated. The best lens model gives an estimate for the distance and mass of the lens via the measurement of the parallax $\pi_{\mathrm{E}}$ using Eq. (1). In Fig. 7 the implied apparent lens brightness based on the mass-magnitude relations and our constraint on the parallax is plotted as a function of lens mass. All bands agree that the lens mass is in the range $0.07<M_{\mathrm{L}} / M_{\odot}<0.10$ with a best estimate of $M_{\mathrm{L}} / M_{\odot}=0.087 \pm 0.010$, preferring a stellar over a sub-stellar host. This is consistent with the previous best estimate of $M_{\mathrm{L}} / M_{\odot}=0.06 \pm 0.04$ (Bennett et al. 2008), but

\footnotetext{
2 http://www . astro.caltech.edu/ jmc/2mass/v3/ transformations/
}
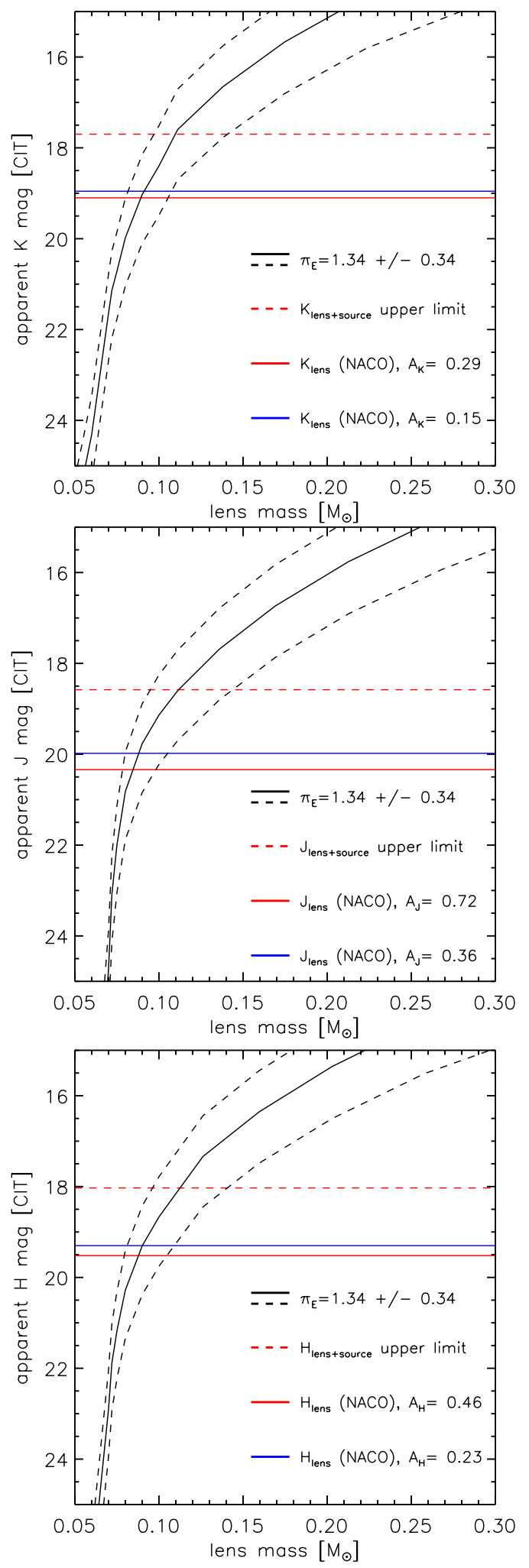

Fig. 7. Mass-Magnitude relations for $K$ (top ), $J$ (middle) and $H$ (bottom) bands [CIT system] derived from Delfosse et al. (2000) for Mdwarfs (with masses $>0.10 M_{\odot}$ ) and L-dwarfs (masses $<0.10 M_{\odot}$ ) from Chabrier et al. (2000). The transition between the two relations at $\sim 0.10 M_{\odot}$ has been linearly interpolated. The black curves show the most likely range of distances for the MOA-2007-BLG-192Lb system as found by Bennett et al. (2008) and the horizontal lines marks the estimate for the lens flux from the NACO data as well as the upper limit of the lens flux from measured lens+source flux for a range of possible interstellar extinctions. 
which was not able to distinguish between the different host star possibilities. This new refined lens mass also affects the inferred planetary mass of MOA-2007-BLG-192Lb. This is due to a light curve degeneracy between the planetary mass ratio $q$, and the source star radius crossing time $t_{*}$. The detection of light from the lens star means that it must be massive enough to be above the hydrogen burning threshold, which constrains $t_{*}<0.05$ days and rules out the cusp crossing models (models I-P of Table 1 in Bennett et al. 2008; the remaining surviving models consistent with the NACO data are listed here in the appendix in Table A.3). This constraint on $t_{*}$ pushes the mass ratio, $q$, toward somewhat smaller values. As a result the range of allowed planetary masses is nearly unchanged.

The physical parameters of the star-planet system can be estimated by the same type of Markov chain Monte Carlo (MCMC) calculations used in Bennett et al. (2008) or Dong et al. (2009). But we now add constraints that the lens star must satisfy the $J H K$ mass luminosity relations of Delfosse et al. (2000), under the assumption that $25 \%$ of the dust responsible for the extinction of the source star is also in the foreground of the lens star plus planet system. The uncertainty in the lens magnitude is taken to be $0.3 \mathrm{mag}$ in each passband. This accounts for the uncertainty in the extinction as well as the uncertainty in the Delfosse et al. (2000) mass-luminosity relations, which become large at low masses because of the metallicity dependence of the minimum stellar mass. The parameter values resulting from this calculation are listed in Table A.2. The planet mass is now $3.2_{-1.8}^{+5.2} M_{\oplus}$, while the host star mass is $0.084_{-0.012}^{+0.015} M_{\odot}$ and the two dimensional star-planet separation during the event is $a=0.66_{-0.22}^{+0.51} \mathrm{AU}$. The MCMC lens distance estimate is $D_{\mathrm{L}}=700_{-120}^{+210} \mathrm{pc}$ which agrees with our more direct estimate of $660_{-70}^{+100}$ pc. This implies that the lens suffers less than half of the total extinction toward the source, and our derived lens colors are consistent with a late $\mathrm{M}$ spectral type (Leggett et al. 2010) of the planetary host.

\subsection{Additional constraints from future high angular resolution observations}

Another improvement can be achieved by measuring the amplitude and direction of the relative proper motion of source and lens in combination with the microlensing modeling of the parallax signal caused by the Earth's motion. Such physical measurements break a model degeneracy in the projected Einstein radius $\tilde{r}_{\mathrm{E}}$ (Bennett et al. 2007, 2008). In the case of MOA-2007BLG-192 the degeneracy is particularly acute because of a gap in event coverage, with different equally well-fitting models requiring widely different projections and hence directions for the relative proper motion, even though the models yield similar am-

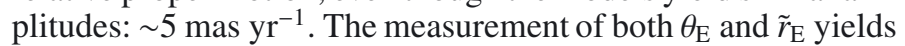
the lens system mass $M_{\mathrm{L}}=c^{2} /(4 G) \theta_{\mathrm{E}} \tilde{r}_{\mathrm{E}}$. Ideally, the relative lens-source proper motion $\mu_{\text {rel }}$ is measured when detecting both the lens star and the source star as done by Alcock et al. (2001).

The two stars will not be fully resolved for many years. However, due to the unique stability of the HST point spread function (PSF) it is possible to measure source-lens separations (with position angles) much smaller than the width of the PSF. This is accomplished by measuring the elongation of the combined lens-source image due to the fact it is a combination of two point source images rather than one. The lens and source stars of MOA-2007-BLG-192 will already be about 25 mas apart in 2012.
Simulations by Bennett et. al. (2007) show that measurements of both the amplitude and orientation are possible for MOA-2007-BLG-192 already in 2012. These measurements combined with our modeling will improve the knowledge of system parameters (masses, orbital separation) to about $10 \%$. The key point is that the direction of the elongation will give us a measurement of the direction of the relative lens-source proper motion $\mu_{\text {rel }}$ and therefore resolve the remaining parallax degeneracies (Ghosh et al. 2004; Bennett et al. 2007, 2008).

\subsection{Properties of the planetary system}

The effective temperature of the planet, for the parameters of the parent star and orbit separation given above, is $47_{-8}^{+7} \mathrm{~K}$ for an albedo of zero, and $40_{-7}^{+6} \mathrm{~K}$ for an albedo of 0.5 . Based on observations of a tenuous atmosphere (20-60 microbars) of nitrogen on Pluto, the temperature of bright surfaces ices on Pluto at perihelion is estimated to be between 35-40 K (Stansberry \& Yelle 1999). Thus, if nitrogen were available, the surface of this planet might look like that of Pluto on the basis of stellar heating alone. However, the large mass of the planet compared with that of Pluto necessitates examining the possible role of heat from the interior of the planet. The maximum temperature possible with zero albedo, $54 \mathrm{~K}$, remains below the pure nitrogen melting point of $63 \mathrm{~K}$, and well below the methane melting point of $91 \mathrm{~K}$.

The present-day terrestrial heat flow $\left(0.087 \mathrm{~W} / \mathrm{m}^{2}\right)$ value is about 10 times less than the roughly $1 \mathrm{~W} / \mathrm{m}^{2}$ deposited by the lensing star on its planet at local noon. Thus the average heat flow coming from the planet itself will not raise the surface temperature significantly, even for a fully rocky body three times the mass of the Earth (Lunine 2010). Of course, we do not know the age of the star; were we to use the Hadean heat flow value for the Earth (Hopkins et al. 2008) for the 3.2 Earth mass body, the influx from geothermal heating could exceed the energy received from the star. The surface temperature could then be above the nitrogen melting point, leading to the possibility of liquid nitrogen lakes or seas if the atmospheric pressure were 0.1 bar or more. The lensing star-planet system is likely older than this, and hence the planet's heat flow correspondingly less.

Because the distribution of heat flow on a terrestrial planet can be strongly heterogeneous, one could imagine places on the surface with much higher heat flow than the average value for the planet, such that temperatures might exceed the melting point not just of nitrogen but of methane. Thus, if sufficient quantities of these molecules were present, the planet's surface might have zones resembling the hydrocarbon lakes and seas of Saturn's moon Titan. The possibility of liquid water cannot be discounted, but would most likely be below the surface or in very restricted, volcanically active, locales.

\section{Conclusions}

In this study we have presented the analysis of photometric data in the near infrared $J H K$ s bands at two different epochs of planetary microlensing event MOA-2007-BLG-192, obtained with the AO system NACO mounted on UT4 at ESO. According to the best-fit lens models as given in Bennett et al. (2008) the difference in the magnification of the source is $0.230 \pm 0.015$ for the two epochs. If the lens is non-luminous this would be the expected photometric gradient in our data set in the absence any blended light contribution. Our $K$-band data, when combined with results from previous optical data, are inconsistent with 
such a scenario at $4,8 \sigma$. In fact the data imply that there is a significant amount of blended light at the location of MOA-2007BLG-192. Assuming that this blend is the lens, the data favor a scenario in which the lens would be a closeby $\left(660_{-70}^{+100} \mathrm{pc}\right)$ late $\mathrm{M}$-dwarf. This is consistent with the estimates for a stellar lens as based on constraints from extended source and parallax effects as discussed in Bennett et al. (2008). While the data available at the time of the discovery paper were consistent with a broad range of planetary host masses, the new NACO data presented here support the hypothesis of a stellar host for MOA-2007-BLG-192Lb. Of course it is conceivable that the detected blend stems not from the lens, but either from a stellar companion to the source, the lens or an unrelated background star. However the probabilities for such scenarios are low and using Ockham's razor the most likely explanation is that the lens is an M-dwarf, which implies a planetary mass of 3.2 $2_{-1.8}^{+5.2} M_{\oplus}$ for MOA-2007-BLG-192Lb, placing it among the front row of known least massive cool planets in orbit around one of the least massive host stars.

MOA-2007-BLG-192Lb is a landmark exoplanet discovery suggesting that planet formation occurs down to to the very low mass end of the stellar population. This is in agreement with the recent statistical results of Cassan et al. (2012) that on average, every star in the Milky Way hosts at least one planet. MOA2007-BLG-192 is the first microlensing event for which multi epoch AO data has been obtained and demonstrates the usefulness of this technique for microlensing, by constraining the physical characteristics of microlensing planetary systems and providing important experiences to optimize future AO observations, which ideally should be carried out in ToO mode for the first epoch, to ensure the source is still significantly magnified.

Acknowledgements. We like to say thanks to the ESO Paranal and Garching teams for their high quality service and support in carrying out the NACO observations. Especially thanks to ESO staff C. Dumas, C. Lidman and P. Amico. Thanks also to the IRSF observatory staff. D.K. and A.C. especially thank ARI, where part of the work was done during a visit. Special thanks to the support from ANR via the HOLMES project grant. Special thanks to David Warren for supporting the work done at Canopus Observatory. D.P.B. was supported by grants NNX07AL71G \& NNX10AI81G from NASA and AST-0708890 from the NSF. Work by S.D. was performed under contract with the California Institute of Technology (Caltech) funded by NASA through the Sagan Fellowship Program. A.G. was supported by NSF grant AST-0757888. J.L. work was financed within the scope of the program "Incentivazione alla mobilita' di studiosi straineri e italiani residenti all'estero". A.U. was supported from the European Research Council under the European Community's Seventh Framework Programme (FP7/2007-2013)/ERC grant agreement No. 246678. D.K. also likes to thank G. James for his graphic support.

\section{References}

Alcock, C., Allsman, R. A., Alves, D. R., et al. 2001, Nature, 414, 617 Beaulieu, J.-P., Bennett, D. P., Fouqué, P., et al. 2006, Nature, 439, 437 Bennett, D. P., \& Rhie, S. H. 1996, ApJ, 472, 660

Bennett, D. P., Anderson, J., Bond, I. A., Udalski, A., \& Gould, A. 2006, ApJ, 647, L171

Bennett, D. P., Anderson, J., \& Gaudi, B. S. 2007, ApJ, 660, 781

Bennett, D. P., Bond, I. A., Udalski, A., et al. 2008, ApJ, 684, 663

Bennett, D. P., Rhie, S. H., Nikolaev, S., et al. 2010, ApJ, 713, 837

Bessell, M. S., \& Brett, J. M. 1988, PASP, 100, 1134

Bond, I. A., Udalski, A., Jaroszyński, M., et al. 2004, ApJ, 606, L155

Cassan, A., Kubas, D., Beaulieu, J.-P., et al. 2012, Nature, 481, 167

Chabrier, G., Baraffe, I., Allard, F., \& Hauschildt, P. 2000, ApJ, 542, 464

Delfosse, X., Forveille, T., Ségransan, D., et al. 2000, A\&A, 364, 217

Devillard, N. 1997, The Messenger, 87, 19

Devillard, N. 1999, in Astronomical Data Analysis Software and Systems VIII, ed. D. M. Mehringer, R. L. Plante, \& D. A. Roberts, ASP Conf. Ser., 172, 333 Diolaiti, E., Bendinelli, O., Bonaccini, D., et al. 2000, A\&AS, 147, 335

Dong, S., Gould, A., Udalski, A., et al. 2009, ApJ, 695, 970

Duquennoy, A., \& Mayor, M. 1991, A\&A, 248, 485

Gaudi, B. S., Bennett, D. P., Udalski, A., et al. 2008, Science, 319, 927

Ghosh, H., DePoy, D. L., Gal-Yam, A., et al. 2004, ApJ, 615, 450

Glass, I. S., \& Nagata, T. 2000, Monthly Notes of the Astronomical Society of South Africa, 59, 110

Gould, A., \& Loeb, A. 1992, ApJ, 396, 104

Gould, A., Udalski, A., An, D., et al. 2006, ApJ, 644, L37

Gould, A., Dong, S., Bennett, D. P., et al. 2010, ApJ, 710, 1800

Hopkins, M., Harrison, T. M., \& Manning, C. E. 2008, Nature, 456, 493

Janczak, J., Fukui, A., Dong, S., et al. 2010, ApJ, 711, 731

Kato, D., Nagashima, C., Nagayama, T., et al. 2007, PASJ, 59, 615

Kubas, D., Cassan, A., Dominik, M., et al. 2008, A\&A, 483, 317

Leggett, S. K., Burningham, B., Saumon, D., et al. 2010, ApJ, 710, 1627

Lenzen, R., Hartung, M., Brandner, W., et al. 2003, in SPIE Conf. Ser. 4841, ed.

M. Iye, \& A. F. M. Moorwood, 944

Liebes, S. 1964, Phys. Rev., 133, 835

Lunine, J. 2010, in Through Time, A Workshop On Titan's Past, Present and Future, ed. V. Cottini, C. Nixon, \& R. Lorenz, 75

Mao, S., \& Paczynski, B. 1991, ApJ, 374, L37

Marigo, P., Girardi, L., Bressan, A., et al. 2008, A\&A, 482, 883

Marshall, D. J., Robin, A. C., Reylé, C., Schultheis, M., \& Picaud, S. 2006, A\&A, 453, 635

Nagayama, T., Nagashima, C., Nakajima, Y., et al. 2003, in SPIE Conf. Ser. 4841, ed. M. Iye, \& A. F. M. Moorwood, 459

Rousset, G., Lacombe, F., Puget, P., et al. 2003, in SPIE Conf. Ser. 4839, ed. P. L. Wizinowich, \& D. Bonaccini, 140

Schechter, P. L., Mateo, M., \& Saha, A. 1993, PASP, 105, 1342

Schlegel, D. J., Finkbeiner, D. P., \& Davis, M. 1998, ApJ, 500, 525

Stansberry, J. A., \& Yelle, R. V. 1999, Icarus, 141, 299

Sumi, T., Bennett, D. P., Bond, I. A., et al. 2010, ApJ, 710, 1641

Udalski, A., Jaroszyński, M., Paczyński, B., et al. 2005, ApJ, 628, L109 


\section{Appendix A: Zeropoints and uncertainties}

Table A.1. Overview of applied calibrations and transformations between the photometric instrumental systems of IRSF and NACO into the 2MASS system.

\begin{tabular}{|c|c|c|}
\hline Band & Photometric calibration & Number of stars used (after last clipping) \\
\hline & IRSF single epoch & \\
\hline$J$ & $J_{\mathrm{IRSF}, 2 \mathrm{MASS}}=22.854 \pm 0.005+J_{\mathrm{IRSF}, \text { inst }}-0.046\left(J_{\mathrm{IRSF}, \text { inst }}-H_{\mathrm{IRSF}, \text { inst }}\right)+0.015$ & 279 \\
\hline$H$ & $H_{\mathrm{IRSF}, 2 \mathrm{MASS}}=22.919 \pm 0.003+H_{\mathrm{IRSF}, \text { inst }}+0.016\left(J_{\mathrm{IRSF}, \text { inst }}-H_{\mathrm{IRSF}, \text { inst }}\right)+0.024$ & 451 \\
\hline$K \mathrm{~s}$ & $K_{\mathrm{IRSF}, 2 \mathrm{MASS}}=22.146 \pm 0.003+K_{\mathrm{IRSF}, \text { inst }}+0.010\left(J_{\mathrm{IRSF}, \text { inst }}-K_{\mathrm{IRSF}, \text { inst }}\right)+0.014$ & 502 \\
\hline & NACO epoch 1 & \\
\hline \multirow{2}{*}{\multicolumn{2}{|c|}{ Zeropoints calibrated against IRSF }} & \\
\hline & & \\
\hline \multicolumn{2}{|c|}{ using star " 1 " (adopted) } & \\
\hline$H$ & $24.012 \pm 0.017+H_{\mathrm{NACO}, \text { inst }}$ & $(24.254 \pm 0.007)$ \\
\hline$K \mathrm{~s}$ & $23.128 \pm 0.017+K_{\mathrm{NACO}, \text { inst }}$ & $\begin{array}{l}(23.987 \pm 0.028) \\
(23.105 \pm 0.020)\end{array}$ \\
\hline \multicolumn{3}{|c|}{ NACO epoch 2} \\
\hline \multirow{2}{*}{\multicolumn{2}{|c|}{$\begin{array}{l}\text { Zeropoints calibrated against IRSF } \\
\text { using star " } 1 "(\text { adopted) }\end{array}$}} & \multirow[b]{2}{*}{ stars “1”+ "2” } \\
\hline & & \\
\hline$J$ & $24.315 \pm 0.018+J_{\mathrm{NACO}, \text { inst }}$ & $(24.345 \pm 0.030)$ \\
\hline$H$ & $24.024 \pm 0.017+H_{\mathrm{NACO}, \text { inst }}$ & $(24.030 \pm 0.060)$ \\
\hline$K \mathrm{~s}$ & $23.067 \pm 0.017+K_{\mathrm{NACO}, \text { inst }}$ & $(23.091 \pm 0.023)$ \\
\hline \multicolumn{3}{|c|}{ Zeropoints aligned with respect to epoch 1 [within $4^{\prime \prime}$ of target] } \\
\hline$J$ & $24.289 \pm 0.019+J_{\mathrm{NACO}, \text { inst }}$ & 8 \\
\hline$H$ & $24.036 \pm 0.012+H_{\mathrm{NACO}, \text { inst }}$ & 10 \\
\hline$K \mathrm{~s}$ & $23.116 \pm 0.008+K_{\mathrm{NACO}, \text { inst }}$ & 10 \\
\hline
\end{tabular}

Notes. The derived zeropoints for NACO are consistent with zeropoints from NACO based on regularly taken standard stars (after correction for atmospheric extinction) and that we do not find a color term between the NACO and 2MASS system.

Table A.2. Parameter values and MCMC uncertainties.

\begin{tabular}{lcc}
\hline \hline Parameter & Value & 2- $\sigma$ range \\
\hline$M$ & $0.084_{-0.012}^{+0.015} M_{\odot}$ & $0.062-0.120 M_{\odot}$ \\
$m$ & $3.2_{-1.8}^{+5.2} M_{\oplus}$ & $0.8-14.8 M_{\oplus}$ \\
$a$ & $0.66_{-0.22}^{+0.51} \mathrm{AU}$ & $0.35-3.17 \mathrm{AU}$ \\
$D_{\mathrm{L}}$ & $0.70_{-0.12}^{+0.21} \mathrm{kpc}$ & $0.5-1.4 \mathrm{kpc}$ \\
$I_{\mathrm{S}}$ & $21.59 \pm 0.07$ & $21.46-21.64$ \\
$q$ & $1.1_{-0.6}^{+1.9} \times 10^{-4}$ & $0.3-5.2 \times 10^{-4}$ \\
\hline
\end{tabular}

Table A.3. Fit parameters for the 8 distinct planetary models for MOA-2007-BLG-192 consistent with the NACO data.

\begin{tabular}{cccccccccccc}
\hline \hline Fit & $\chi^{2}$ & $t_{\mathrm{E}}$ & $t_{0}^{\prime}$ & $u_{0}$ & $d$ & $\theta$ & $q$ & $t_{*}$ & $I_{\mathrm{s}}$ & $\pi_{\mathrm{E}}$ & $\phi_{\mathrm{E}}$ \\
\hline A & 1121.12 & 82.5 & 5.442 & 0.00309 & 0.912 & $236.9^{\circ}$ & $3.7 \times 10^{-5}$ & 0.040 & 21.61 & 1.51 & $208.5^{\circ}$ \\
B & 1121.16 & 83.2 & 5.442 & 0.00306 & 1.120 & $236.8^{\circ}$ & $3.7 \times 10^{-5}$ & 0.041 & 21.62 & 1.49 & $208.7^{\circ}$ \\
C & 1121.66 & 82.9 & 5.456 & -0.00349 & 0.807 & $105.5^{\circ}$ & $3.4 \times 10^{-4}$ & 0.041 & 21.60 & 1.47 & $209.2^{\circ}$ \\
D & 1122.08 & 83.5 & 5.448 & -0.00296 & 1.113 & $121.9^{\circ}$ & $5.8 \times 10^{-5}$ & 0.041 & 21.62 & 1.44 & $209.8^{\circ}$ \\
E & 1125.41 & 83.2 & 5.454 & 0.00306 & 0.890 & $240.1^{\circ}$ & $7.6 \times 10^{-5}$ & 0.048 & 21.61 & 1.19 & $337.5^{\circ}$ \\
F & 1125.44 & 82.5 & 5.454 & 0.00309 & 1.118 & $239.9^{\circ}$ & $7.5 \times 10^{-5}$ & 0.049 & 21.60 & 1.16 & $332.3^{\circ}$ \\
G & 1125.48 & 81.8 & 5.450 & -0.00313 & 0.897 & $120.3^{\circ}$ & $6.4 \times 10^{-5}$ & 0.049 & 21.60 & 1.13 & $336.5^{\circ}$ \\
H & 1125.50 & 80.8 & 5.450 & -0.00309 & 1.110 & $120.6^{\circ}$ & $6.1 \times 10^{-5}$ & 0.048 & 21.61 & 1.19 & $337.8^{\circ}$ \\
\hline
\end{tabular}

Notes. $t_{0}^{\prime}=t_{0}-4240$ days. $t_{0}$ and $u_{0}$ are the time and distance of the closest approach of the source to the lens center-of-mass. $q$ and $d$ are the planet: star mass ratio and separation, and $\theta$ is the angle between the source trajectory and the planet-star axis. $I_{\mathrm{s}}$ is the best-fit source magnitude, and $\pi_{\mathrm{E}}$ and $\phi_{\mathrm{E}}$ are the magnitude and angle of the microlensing parallax vector. The units for the Einstein radius crossing time, $t_{\mathrm{E}}$, the source radius crossing time, $t_{*}$, and $t_{0}^{\prime}$ are days, and all other parameters are dimensionless. 